\title{
The effect of COVID-19 on essential surgical admissions in South Africa: A retrospective observational analysis of admissions before and during lockdown at a tertiary healthcare complex
}

J Moustakis, ${ }^{1}$ MB BCh; A A Piperidis, ${ }^{2}$ BEngSc, MB BCh; A B Ogunrombi, ${ }^{2,3}$ MBBS, FWACS, MSc (Med) (Cardiothoracic Surgery)

${ }^{1}$ Johannesburg District Health, Gauteng Department of Health, Johannesburg, South Africa

${ }^{2}$ Klerksdorp-Tshepong Hospital Complex, Klerksdorp, South Africa

${ }^{3}$ Department of Surgery, Faculty of Health Sciences, University of the Witwatersrand, Johannesburg, South Africa

Corresponding author: J Moustakis (moustakisy@gmail.com)

\begin{abstract}
Background. With COVID-19 having spread across the globe, it has become standard to implement infection control strategies (colloquially known as lockdown) with the intention of reducing the magnitude and delaying the peak of the epidemiological curve. Personal infection mitigation strategies coupled with lockdown have caused a change in healthcare-seeking behaviour, with individuals not attending to their ill health as they previously did.

Objectives. To determine whether admissions for urgent and emergency surgical pathologies have declined during the COVID-19 lockdown period, and the magnitude of the decline.

Methods. A retrospective analysis was conducted, comparing pre-lockdown (3 February - 26 March 2020) and lockdown (27 March 30 April 2020) admission incidences for surgical pathologies at a tertiary healthcare complex in North West Province, South Africa. Poisson regression models were created to determine admission incidence rate ratios (IRRs).

Results. Of 769 surgical admissions included in the analysis, $49.7 \%$ were male and $67.2 \%$ were unemployed. There was a $44 \%$ reduction in the incidence of non-trauma admissions during lockdown (IRR 0.56; $95 \%$ confidence interval (CI) $0.47-0.68 ; p<0.001$ ) and a $53 \%$ reduction in the incidence of trauma-related admissions (IRR 0.47; $95 \%$ CI $0.34-0.66 ; p<0.001$ ).

Conclusions. Even when the prevalence of SARS-CoV-2 infection was minimal, COVID-19 lockdown in North West was associated with a significant reduction in surgical admissions. In order to ensure an overall benefit to public health, a balance between maintaining the integrity of COVID-19 control mechanisms and access to healthcare services is essential.
\end{abstract}

S Afr Med J 2020;110(9):910-915. https://doi.org/10.7196/SAMJ.2020.v110i9.15025

SARS-CoV-2, the pathogen that causes COVID-19, was initially identified on 31 December 2019 in Wuhan City, Hubei Province, China. Thereafter, the virus spread across the globe, with the first case in South Africa (SA) confirmed on 5 March 2020 in KwaZuluNatal Province. ${ }^{[1]}$

The President of SA declared a 3-week nation-wide lockdown, starting on 27 March 2020, in an effort to reduce the transmission rate, a decision guided by the Ministerial Advisory Committee on COVID-19. ${ }^{[2]}$ The lockdown was subsequently extended to 1 May 2020, after which phased easing of restrictions would occur at a rate directed by epidemiological trends and healthcare operational capacity. ${ }^{[3]}$

At its highest alert level, the lockdown regulated personal movement by implementing a curfew and mandating the public to remain at home other than for the purposes of purchasing essential living supplies and seeking emergency or chronic medical attention. ${ }^{[4]}$ Furthermore, transport regulations limited the operating hours and carrying capacity of public transport vehicles. ${ }^{[3]}$ It is important to note the personal financial cost that would result from these measures, especially in the context of SA, where poverty is rife and the unemployment rate is $29 \%$, making the population vulnerable to economic instability. ${ }^{[5]}$

Additionally, the general public was inundated with news reports stressing the severity of COVID-19 and social media trends, instantiated by \#StayAtHome, highlighting the importance of social distancing.

Ultimately these measures resulted in people ensconcing themselves in their homes and specifically avoiding healthcare facilities, which are perceived as places of high transmission risk, notwithstanding the National Department of Health's efforts to reduce this danger. ${ }^{[6]}$ Many people's finances have been depleted, raising the question of affordability of the already lockdown-limited public transport system. Our hypothesis is that these inadvertent outcomes prevent patients from accessing healthcare, either physically owing to inaccessible transport, or psychologically through the fear of COVID-19 prompting personal infection mitigation behaviours.

\section{Objectives}

Having speculatively considered reasons driving a possible decline in admission statistics, we endeavoured through this research to investigate the change in urgent and emergency admissions to the Department of Surgery at Klerksdorp-Tshepong Hospital Complex (KTHC) prior to and during the first stage of COVID-19 lockdown.

\section{Methods}

Setting

KTHC is a tertiary hospital in the largest city of North West Province, $\mathrm{SA}$, and provides partial level 3 services to the entire province. ${ }^{[7]}$ So 
far, the province has been relatively spared from the pandemic, the first cases of COVID-19 being confirmed on 21 March 2020, reaching 31 cases by the end of the study period (approximately 1 case per 130000 population). ${ }^{[8,9]}$

Prior to the commencement of lockdown, no changes to the admission and referral criteria of the hospital complex were implemented. From 27 March 2020, KTHC cancelled most elective admissions, but continued to accept referrals and admit patients with urgent and emergency pathologies as usual. During lockdown, the decision as to which surgical services are offered has been guided by KTHC COVID-19 bed occupancy. When bed pressure is low ( $<30 \%$ of COVID-19 beds occupied), emergency, urgent and semiurgent elective procedures are performed. COVID-19 bed occupancy levels of $30-60 \%$ will allow for emergency and urgent procedures. Once bed occupancy is $>60 \%$, only emergency admissions will be considered.

\section{Study design}

An observational, retrospective analysis of surgical admissions to KTHC was conducted. The research population included patients aged $\geq 18$ years admitted to the Department of Surgery from 3 February to 30 April 2020. A comparison of admission statistics was performed over a period of 53 days prior to COVID-19 lockdown (control group) and a 35-day period during lockdown (exposed group).

\section{Participants}

Not all hospital admissions are for context-relevant essential services, as defined by the World Health Organization, ${ }^{[10]}$ so any hypothetical drop in admission numbers during lockdown could be explained by loss of patients seeking non-essential services.

A proxy for determining inappropriate decreases in admissions (loss of patients requiring essential care) would be a change in the number of admissions for pathologies that:

- have incidences that are unlikely to change precipitously or due to a known pathophysiological mechanism causally related to COVID-19

- confer serious risks to health

- require interventions where the rate of success is time-sensitive.

Using the above criteria, the data were refined to include only the following diagnoses: acute abdomen (all causes), bowel obstruction (all causes), critical and acute limb ischaemia, appendicitis, upper gastrointestinal bleeding, soft-tissue infections, and management of suspected or confirmed malignancies. Trauma-related admission statistics were also acquired, but there is a caveat to their interpretation: the incidence of trauma is likely to be directly reduced by lockdown, limiting its use for determining inappropriate decreases in admissions.

Throughout the study period, COVID-19 bed occupancy at KTHC did not exceed $30 \%$, so admissions and referrals of the studied pathologies were not restricted by policy.

\section{Statistical analysis}

Admission data were dichotomised into non-trauma and traumarelated admissions. Count data were captured as admissions per day, holding the denominator, which constitutes the population residing in the hospital's catchment area, as constant throughout the sampling time. These data were descriptively analysed and summarised.

Two univariate Poisson regression models were fitted to determine the incidence rates and incidence rate ratios (IRRs) for the dichotomised data: total admissions, modelled as the response variable, and the explanatory variable constituting the date, which was factorised into two levels, pre-lockdown or during COVID-19 lockdown. Thereafter, the Poisson regression analyses were stratified according to diagnosis, age, sex and employment status to allow for the evaluation of interactions and possible high-risk groups identified a priori (female sex, the unemployed and the elderly).

Admissions with missing employment status, of which there were 156 (20.3\%), were included as a third category (unknown) in the subgroup analysis.

All confidence levels were set to $95 \%(\alpha=0.05)$, and Wald 95\% confidence intervals (CIs) were reported.

\section{Secondary analysis}

A secondary analysis on admissions for appendicitis was conducted. Pre-lockdown and lockdown differences in mean 'time to intervention' were compared using a two-sample, two-tailed $t$-test. Changes in severity of appendicitis were assessed using ordinal logistic regression.

The following definitions were applied:

- Time to intervention: time (hours) from admission to appendicectomy (non-operative management of appendicitis is rarely conducted at the facility)

- Severity: graded from intraoperative notes, as described by Gomes et al. ${ }^{[11]}$

Missing appendicitis-related observations occurred when patient records were not found or the required information was not recorded. The missingness mechanism was assessed as missing completely at random (confirmed by Little's $\chi^{2}$ test). Multiple imputation analysis was incorporated to account for missing observations. Time to intervention had 5 missing observations (14.7\%), and predictive mean matching was used to impute complete data sets. Severity of appendicitis had 4 missing observations (11.8\%), and a proportional odds logistic regression model was used to impute complete data sets.

\section{Ethical considerations}

Ethics approval was granted by the University of the Witwatersrand Human Research Ethics Committee (ref. no. M200526) and the Patient Safety Group of KTHC.

\section{Results}

There was a total of 1512 admissions during the study period (1 109 during pre-lockdown and 403 during lockdown). These were refined to 769 admissions (567 during pre-lockdown and 202 during lockdown) once patients not specified in the design were excluded (such as elective admissions and patients aged $<18$ years).

\section{Non-trauma admissions}

A total of 579 patients were admitted to the Department of Surgery for specified non-trauma-related pathologies during the study period: $67.2 \%$ of the study population were unemployed, $49.7 \%$ were male, and the average age was 52.5 years. Table 1 summarises the patient characteristics. There was a $44 \%$ reduction in non-trauma admissions during the COVID-19 lockdown period, from 7.96 admissions per day pre-lockdown to 4.49 admissions per day during lockdown (IRR 0.56; 95\% CI 0.47 - 0.68; $p<0.001$ ) (Fig. 1).

Management of suspected or confirmed malignancy was the most common reason for admission during the study period, accounting for $45.9 \%$ of total admissions. The admission incidence for this subgroup declined by $31 \%$ (IRR 0.69 ; 95\% CI 0.53 - 0.89) during the lockdown period. The most pronounced reduction in admission incidence was noted for admissions for upper gastrointestinal bleeding, which 
Table 1. Summary of characteristics of patients admitted for specified surgical pathology during the study period ${ }^{*}$

\begin{tabular}{|c|c|c|c|}
\hline & Total $(N=769)$ & Pre-lockdown $^{\dagger}(N=567)$ & Lockdown $^{*}(N=202)$ \\
\hline Non-trauma, $N$ & 579 & 422 & 157 \\
\hline Age (years), mean (SD) & $52.5(16.7)$ & $52.9(17)$ & $55(15.8)$ \\
\hline Sex (male), $n(\%)$ & $288(49.7)$ & $204(48.3)$ & $84(53.5)$ \\
\hline \multicolumn{4}{|l|}{ Employment status, $n(\%)^{\varsigma}$} \\
\hline Employed & $69(11.9)$ & $49(11.6)$ & $20(12.7)$ \\
\hline Unemployed & $389(67.2)$ & $293(69.4)$ & $96(61.1)$ \\
\hline \multicolumn{4}{|l|}{ Diagnosis, $n(\%)$} \\
\hline Appendicitis & $34(5.9)$ & $26(6.2)$ & $8(5.1)$ \\
\hline Acute abdomen & $15(2.6)$ & $11(2.6)$ & $4(2.5)$ \\
\hline Bowel obstruction & $50(8.6)$ & $36(8.5)$ & $14(8.9)$ \\
\hline Acute and critical limb ischaemia & $23(4.0)$ & $16(3.8)$ & $7(4.5)$ \\
\hline Soft-tissue infection & $135(23.3)$ & $104(24.6)$ & $31(19.7)$ \\
\hline Malignancy (suspected and confirmed) & $266(45.9)$ & $183(43.4)$ & $83(52.9)$ \\
\hline Upper gastrointestinal bleeding & $56(9.7)$ & $46(10.9)$ & $10(6.4)$ \\
\hline Trauma, $N$ & 190 & 145 & 45 \\
\hline Age (years), mean (SD) & $33.7(12.8)$ & $33.1(11.9)$ & $36(15.5)$ \\
\hline Sex (male), $n(\%)$ & $155(81.6)$ & $116(80.0)$ & $39(86.7)$ \\
\hline \multicolumn{4}{|l|}{ Employment status, $n(\%)^{\varsigma}$} \\
\hline Employed & $27(14.2)$ & $22(15.1)$ & $5(11.1)$ \\
\hline Unemployed & $128(67.4)$ & $101(69.7)$ & $27(60.0)$ \\
\hline
\end{tabular}

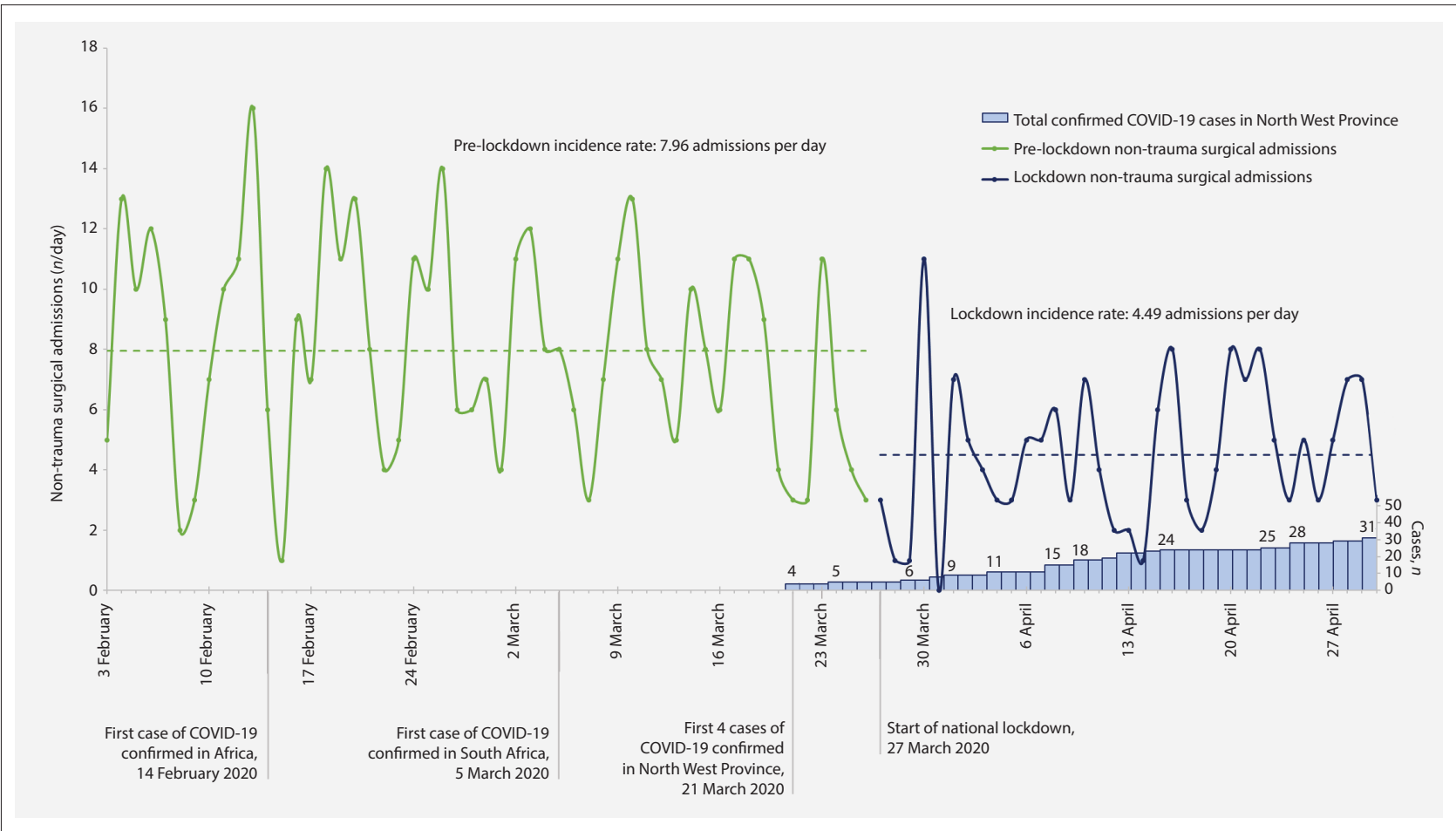

Fig. 1. Daily non-trauma surgical admissions and cumulative COVID-19 cases in North West Province, South Africa. The pre-lockdown period constitutes the time from 3 February 2020 to the day before national lockdown (26 March). The lockdown period constitutes the days from 27 March to 30 April. Incidence rates were calculated by dividing the number of cumulative admissions in each period by the number of days in each period. Key developments are noted below the axis.

contributed to $9.7 \%$ of total admissions. The reduction in admission incidence during lockdown was 67\% (IRR 0.33; 95\% CI 0.17 - 0.65). Both male and female sexes experienced a decline in admissions during lockdown, at $38 \%$ (IRR 0.62; 95\% CI $0.48-0.80$ ) and $49 \%$ (IRR 0.51; 95\% CI 0.39 - 0.66), respectively.
There was no significant deviation from the cumulative result when stratified by employment status and age. Fig. 2 illustrates the results of the complete subgroup analysis conducted on non-trauma admissions. No adjustments were made to the $95 \%$ CIs for multiple hypothesis testing. 


\section{Trauma-related admissions}

Of the 190 patients admitted due to trauma, the majority were male (81.6\%). The average age was 33.7 years (Table 1). There was a $53 \%$ reduction in trauma-related admissions during the COVID-19 lockdown period, from 2.74 admissions per day pre-lockdown to 1.29 during lockdown (IRR 0.47; 95\% CI $0.34-0.66 ; p<0.001)$.

When sex-based subgroups were analysed, male trauma-related admissions declined by $49 \%$ during lockdown (IRR $0.51 ; 95 \%$ CI 0.35 - 0.73), compared with a $69 \%$ decline for females (IRR $0.31 ; 95 \%$ CI 0.13 - 0.75).

Fig. 3 illustrates the results of the complete subgroup analysis conducted on trauma admissions. No adjustments were made to the $95 \%$ CIs for multiple hypothesis testing.

\section{Secondary analysis of appendicitis}

A total of 34 admissions were due to appendicitis, 26 during the pre-lockdown period and 8 during lockdown. The mean (standard deviation) age of the patients was 32.2 (11.5) years, and $55.9 \%$ were male. The time to appendicectomy decreased from an average of 23.29 hours pre-lockdown to 14.25 hours during COVID-19 lockdown (mean difference 9.04 hours; 95\% CI -1.46 19.54; $p=0.09)$. There was no significant difference in the severity of appendicitis, and lockdown was not independently associated with an increase in the intraoperative grading of appendicitis (OR 1.19; 95\% CI 0.262 5.417; $p=0.94)$.

\section{Discussion}

This study examined the effect of COVID19 and the accompanying lockdown on admissions of surgical pathologies at KTHC, North West, SA.

The decrease in non-trauma admissions during lockdown is probably multifactorial in origin, with contributing factors including the public exhibiting risk-aversion behaviour elicited by fear of contracting COVID-19, restrictions on personal movement, and financial constraints limiting access to transport.

The reduction in trauma admissions has an additional potential influence from the causal relationship between certain lockdown implements (specifically the ban on alcohol sales, restrictions on personal movement and increased police presence) and the incidence of interpersonal violence and vehicular accidents (both major contributors to trauma incidence)

In the non-trauma subgroup analysis, the effect of COVID-19 was observed across all age groups and in both the employed and unemployed with similar magnitude.

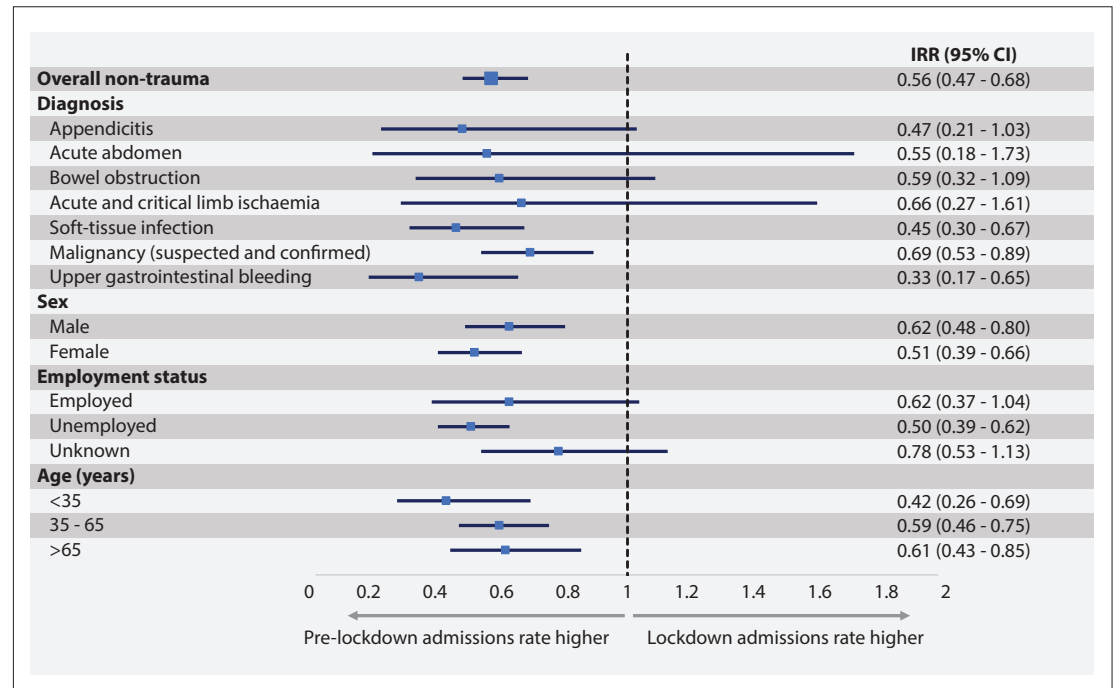

Fig. 2. Incidence of non-trauma admissions (n/day), comparing the pre-lockdown period (3 February - 26 March 2020) with the lockdown period (27 March - 30 April) in prespecified subgroups. No adjustments for multiple testing have been made to the $95 \%$ CIs, which should therefore be interpreted cautiously to infer definitive effects. (CI = confidence interval; $I R R=$ incidence rate ratio.)

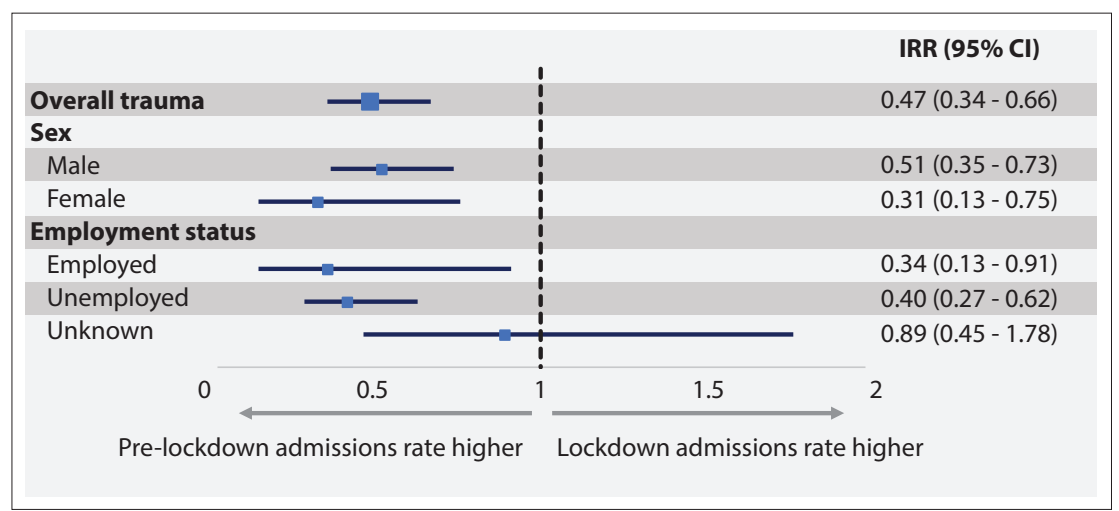

Fig. 3. Incidence of trauma admissions (n/day), comparing the pre-lockdown period (3 February 26 March 2020) with the lockdown period (27 March - 30 April) in prespecified subgroups. No adjustments for multiple testing have been made to the 95\% CIs, which should therefore be interpreted cautiously to infer definitive effects. (CI = confidence interval; $I R R=$ incidence rate ratio.)

When stratified by diagnosis, the reduction was most pronounced in admissions for upper gastrointestinal bleeding, which could be the result of a pathophysiological contributory relationship between the ban on the sale of alcohol and a true reduction in the incidence of upper gastrointestinal bleeding in the population. ${ }^{[12,13]}$ Female sex (identified as a vulnerable subgroup $a$ priori), in both trauma- and non-traumarelated admissions, was associated with a greater reduction in admission incidence than male sex.

However, owing to the reduced statistical power and established weaknesses of subgroup analyses, we cannot infer definitive differences within the subgroups, and the above interpretation should be considered with this in mind.

It was originally hypothesised that personal and government-regulated infection control measures would prevent patients from attending to their health expeditiously, resulting in delayed presentations with more severe disease. This was not observed in the study population, where no difference was noted in severity of appendicitis between the pre-lockdown and lockdown groups. We did observe a decrease in time from admission to appendicectomy, probably owing to fewer patients utilising surgical services, as demonstrated in the primary analysis, and so reducing theatre waiting times. Although the severity of appendicitis has many determinants, a possible explanation for the unexpected equivalence of appendicitis severity between the groups is the quicker time to theatre; however, the sample size was unexpectedly small, so meaningful inferences from this analysis could not be made.

What is alarming, when considering the results, is the magnitude of the reduction in 
admission incidence rates in a population with a low prevalence of COVID-19. The SA response to the pandemic was aggressive, with national lockdown being implemented only 6 days after the first cases were confirmed in North West. This prompt reaction slowed the spread of SARS-CoV-2 infection, but may have contributed to the exaggerated decrease in admission rates. The benefit of the infection control strategies in such a situation may be outweighed by the cost to public health, as individuals with pathologies that carry a significant risk to wellbeing are not presenting to healthcare facilities.

The decline in hospital admissions was initially welcomed and granted a reprieve to prepare for the potential surge of patients by liberating resources, both human and financial, for reallocation to COVID-19 infection prevention and management. However, concerns are being raised regarding the overall consequences to public health as the outcomes of the unaccounted-for admissions are considered. Admissions for work-up of suspected malignancy and management of confirmed malignancy decreased by $31 \%$ during lockdown. This could translate to delays in the diagnosis and management of malignancy, a pathology where such delays may have deleterious consequences in terms of prognosis. Although beyond the scope of this investigation, this has been demonstrated to be the case in the Netherlands, where fewer cancer diagnoses were being made during the COVID-19 pandemic. ${ }^{[14]}$

Admissions for appendicitis decreased by 53\% during lockdown. The natural history of appendicitis is well established, ${ }^{[15]}$ with the most severe outcome of untreated disease being perforation that may lead to death if peritonitis develops. It is expected that some of the patients who did not seek medical care may have died as a result of their illnesses, resulting in an increase in mortality statistics. When reviewing the data on weekly deaths released by the South African Medical Research Council, the expected increase in natural (nontrauma) deaths during the studied part of the lockdown period is not apparent. ${ }^{[16]}$ However, interpretation of the results is challenging, as the lockdown implements may interfere with the spread of other communicable diseases, decreasing non-COVID-19 infections and so reducing natural-cause mortality. This explanation would be supported by evidence that in populations with a lower burden of communicable disease there has been an excess in natural-cause mortality during lockdown that is not attributable to COVID-19, as is the case in England and Wales. ${ }^{[17]}$

In SA, excess natural deaths have manifested beyond the study period, peaking during the week of 21 July 2020. ${ }^{[16]}$ A significant number of deaths are not accounted for by confirmed COVID-19 fatalities; this may be due to under-reporting of COVID-19 deaths and possibly, as hypothesised, to a reduction in healthcare accessibility leading to deaths that would have normally been prevented. Further research is required to objectively clarify the uncertainties surrounding excess mortality during the COVID-19 pandemic.

COVID-19 has had consequences that have spread throughout the world and influenced all divisions of medicine, while at the same time providing opportunities to explore previously uninvestigated hypotheses. Further research is required to completely elucidate the possible interactions uncovered, such as the role of the ban on alcohol sales and other lockdown implements on the incidence of certain pathologies such as upper gastrointestinal bleeding and trauma. If our hypothesis is correct, as lockdown restrictions are eased there will be a disproportionate stepwise increase in the admission incidences of such pathologies.

\section{Study limitations}

The study was limited in its ability to determine how the spread of COVID-19 in the community influenced admission rates without additional effects from lockdown measures, as the two events occurred in rapid succession. Furthermore, the analysis was restricted to a single healthcare complex where the majority of patients were unemployed, thereby making external generalisability limited. Particularly in populations with higher levels of employment and income, decreases in admission incidences may be the result of patients moving from state to private healthcare. However, similar findings have been reported for admissions for acute coronary syndrome in dissimilar populations in Northern Italy and California, USA. ${ }^{[18,19]}$

Seasonal variation is a well-recognised phenomenon in respiratory and cardiovascular disease. It may also play a role in the incidence of some surgical pathologies. ${ }^{[20-23]}$ However, when surgical admissions are observed as a whole, a typical seasonal relationship has not been established. ${ }^{[24]}$ Klerksdorp has a temperate climate pattern, with the study period running through late summer and early autumn. When considering the diagnosis-subgrouped analysis and trauma-related admissions, meteorological factors, which have not been controlled for, may contribute to some of the variation observed. It would be pertinent to include additional date-matched control periods for further research to attempt to control for seasonal variation.

\section{Conclusions}

This research confirmed that there was a substantial reduction in the admission incidence of surgical pathologies that require urgent or emergency interventions at the healthcare complex studied. Solutions to improve healthcare access must be devised and implemented, while simultaneously maintaining the integrity of the COVID-19 control mechanisms.

\section{Declaration. None.}

\section{Acknowledgements. None.}

Author contributions. JM: conception, study design and statistical analysis; AAP: conception, study design and data acquisition; ABO: study design. Funding. None.

Conflicts of interest. None.

1. National Department of Health, South Africa. First case of COVID-19 coronavirus reported in SA. 5 March 2020. http://www.health.gov.za/index.php/2014-03-17-09-48-36/2014-03-17-09-4950? download=3892:media-statement-first-case-of-covid-19-coronavirus-reported-in-sa-04-march2020\&start=135 (accessed 7 June 2020).

2. Abdool Karim S. The South African response to the pandemic. N Engl J Med 2020;382:e95. https:// doi.org/10.1056/nejmc2014960

3. National Department of Health, South Africa. COVID-19 Online Resource and News Portal. National Department of Health, South Africa. COVID-19 Online Resource and News Portal.
COVID-19 risk adjusted strategy. 28 May 2020. https://sacoronavirus.co.za/covid-19-risk-adjustedCOVID-19 risk adjusted strategy.
strategy/ (accessed 7 June 2020).

4. South African Government. Disaster Management Act, 2002 (Act No. 57 of 2002). Regulations to address, prevent and combat the spread of coronavirus COVID-19: Amendment. Government Gazette No. 43148, 25 March 2020. (Published under Government Notice R398.) https://www.gov.za/sites/ default/files/gcis_document/202003/4314825-3cogta.pdf (accessed 7 June 2020).

5. Statistics South Africa. Unemployment rises slightly in third quarter of 2019. 29 October 2019. http:// www.statssa.gov.za/?p=12689 (accessed 7 June 2020).

6. Wong L, Hawkins J, Langness S, Murrell K, Iris P, Sammann A. Where are all the patients? Addressing Covid-19 fear to encourage sick patients to seek emergency care. N Engl J Med (epub 14 May 2020). https://catalyst.nejm.org/doi/full/10.1056/CAT.20.0193 (accessed 5 June 2020).

Klerksdorp Tshepong Hospital Complex. https://www.klerksdorptsheponghospital.org/about-us/ (accessed 8 June 2020).

8. Statistics South Africa. Mid-year population estimates, 2020. http://www.statssa.gov.za/?page id $=1854 \&$ PPN=P0302 (accessed 8 June 2020).

9. South African Government. MEC Madoda Sambatha: Status of coronavirus in the North West. https:// www.gov.za/speeches/north-west-province-24-mar-2020-0000 (accessed 8 June 2020).

10. World Health Organization. COVID-19: Operational guide for maintaining essential health services during outbreak: Interim guidance, 25 March 2020. https://apps.who.int/iris/handle/10665/331561 (accessed 5 June 2020).

11. Gomes CA, Sartelli M, Di Saverio S, et al. Acute appendicitis: Proposal of a new comprehensive grading system based on clinical, imaging and laparoscopic findings. World J Emerg Surg 2015;10:60. https:// doi.org/10.1186/s13017-015-0053-2

12. Kärkkäinen J, Miilunpohja S, Rantanen T, et al. Alcohol abuse increases rebleeding risk and mortality in patients with non-variceal upper gastrointestinal bleeding. Dig Dis Sci 2015;60(12):3707-3715. https://doi.org/10.1007/s10620-015-3806-6

13. Kelly JP, Kaufman DW, Koff RS, Laszlo A, Wiholm BE, Shapiro S. Alcohol consumption and the risk of major upper gastrointestinal bleeding. Am J Gastroenterol 1995;90(7):1058-1064

14. Dinmohamed A, Visser O, Verhoeven R, et al. Fewer cancer diagnoses during the COVID-19 epidemic in the Netherlands. Lancet Oncol 2020;21(6):750-751. https://doi.org/10.1016/s1470-2045(20)30265-5 
15. Temple CL, Huchcroft SA, Temple WJ. The natural history of appendicitis in adults: A prospective study. Ann Surg 1995;221(3):278-281. https://doi.org/10.1097/00000658-199503000-00010

16. South African Medical Research Council. Report on weekly deaths in South Africa: 1 January 23 June 2020. https://www.samrc.ac.za/reports/report-weekly-deaths-south-africa?bc=254 (accessed 8 June 2020).

17. Griffin S. Covid-19: 'Staggering number' of extra deaths in community is not explained by Covid-19. BMJ 2020;369:m1931. https://doi.org/10.1136\%2Fbmj.m1931

18. Solomon M, McNulty E, Rana J, et al. The Covid-19 pandemic and the incidence of acute myocardial infarction. N Engl J Med 2020 (epub 19 May 2020). https://doi.org/10.1056/nejmc2015630

19. De Filippo O, D’Ascenzo F, Angelini F, Bocchino P, Conrotto F, Saglietto A. Reduced rate of hospital admissions for ACS during Covid-19 outbreak in northern Italy. N Engl J Med 2020;383:88-89. https:// doi.org/10.1056/nejmc2009166

20. Zangbar B, Rhee P, Pandit V, et al. Seasonal variation in emergency general surgery. Ann Surg 2016;263(1):76-81. https://doi.org/10.1097/sla.0000000000001238
21. Milford KL, Navsaria PH, Nicol AJ, Edu S. Trauma unit attendance: Is there a relationship with weather, sporting events and week/month-end times? An audit at an urban tertiary trauma unit in

2. Zimmerman J, Arnon R, Beeri R, et al. Seasonal fluctuations in acute upper gastrointestinal bleedin Lack of effect of nonsteroidal anti-inflammatory drugs. Am J Gastroenterol 1992;87(11):1587-1590. 23. Miller R, Pywell S, Chan J, McCluney S, Halsey T. Seasonal variation in hand and upper limb soft tissue infections. J Hand Surg Asian Pac Vol 2019;24(2):189-194. https://doi.org/10.1 142/s2424835519500255 24. Fullerton KJ, Crawford VL. The winter bed crisis-quantifying seasonal effects on hospital bed usage. Q J Med 1999;92(4):199-206. https://doi.org/10.1093/qjmed/92.4.199

Accepted 13 July 2020 